\title{
TEMA 7-2016: Rehabilitación en VIH/SIDA
}

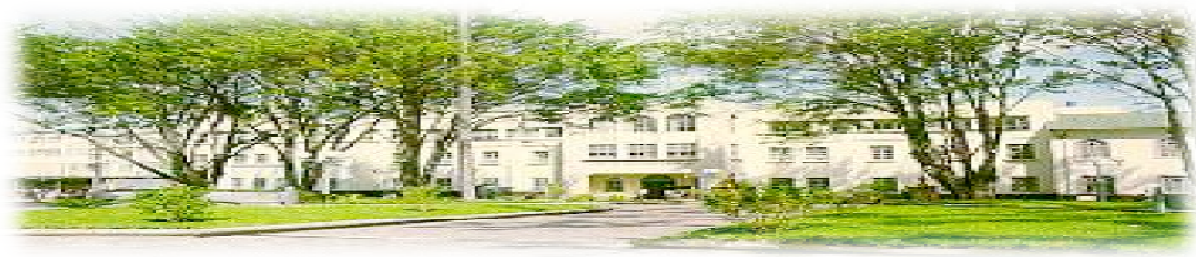

Hospital San Juan de Dios, San José, Costa Rica. Fundado en 1845
Recibido:
$20 / 11 / 2015$
Aceptado:
$03 / 12 / 2015$

Vanessa Uclés Villalobos ${ }^{1}$

Roison Ali Espinoza Reyes ${ }^{2}$

${ }^{1}$ Médico Fisiatra. Msc. En Cuidados Paliativos del servicio de Rehabilitación del Hospital Rafael Ángel Calderón Guardia. Caja Costarricense de Seguro Social.Correo electrónico: dravanessaucles@gmail.com

${ }^{2}$ FisioterapeutaEspecialista en Discapacidad y políticas públicas del servicio de Rehabilitación del Hospital Rafael Ángel Calderón Guardia. Caja Costarricense de Seguro Social. Correo electrónico: roison ali@hotmail.com

\section{RESUMEN}

Los primeros casos reportados de VIH remontan a la fecha de 1983, desde entonces se ha desarrollado una pandemia de la infección a nivel mundial. Siendo esta una de las enfermedades emergentes que han incrementado en los últimos años y Costa Rica no escapa a esta tendencia.

Los efectos adversos a los medicamentos, las infecciones oportunistas y la sintomatología del HIV hacen que ésta población quede predispuesta a la limitación de sus capacidades funcionales. Los efectos secundarios a los anti retrovirales generan toxicidad que alteran la homeostasis del organismo creando paso a una serie de síndromes y cuadros clínicos que afectan al aparato locomotor como lo son neuropatías, lipohipertrofia, lipoatrofia, miopatías, entre otras.
La rehabilitación puede ser ampliamente definida como cualquiera de los servicios y actividades que tratan o previenen deficiencias, limitaciones en la actividad diaria o participaciones de restricciones experimentadas por un individuo, cuyos beneficios podrían satisfacer y ayudar a contribuir con el bien estar físico, emocional, social, laboral y funcional de una persona creando una autonomía en la dependencia de las actividades diarias o adaptaciones para los pacientes que así lo requieran.

\section{PALABRAS CLAVE}

Rehabilitación, VIH, SIDA 


\section{ABSTRACT}

The first cases of HIV reported date to 1983 have since, develop a pandemic infection worldwide. $\mathrm{HIV}$ is one of the emerging diseases that have been increased in years. Costa Rica is no exception

The adverse effects to medications, opportunistic infections, and symptoms of HIV, make the population predisposed to limitation of their functional abilities. The adverse effects to antiretroviral treatment generates toxicity that alters of homeostasis of the organism, which consequently creates a number of syndromes and clinical conditions. Furthermore, those affects the musculoskeletal system as in neuropathies, lipohypertrophy, lipoatrophy, myopathies, among others diseases.

Rehabilitation can be broadly defined as any services and activities that treat, or prevent deficiencies, limitations on daily activities. Or participation restrictions, experienced by an individual, who benefits could meet and help contribute to the physical wellbeing functional and emotional status, or social or adaptations for patients who require it.

\section{KEY WORDS}

Rehabilitation, HIV, AIDS

\section{INTRODUCCIÓN}

En Costa Rica, se considera que se deben continuar generando oportunidades para el mejoramiento de la calidad de vida de quienes viven con virus de inmunodeficiencia humana $\mathrm{y} / \mathrm{o}$ síndrome de inmunodeficiencia adquirida(VIH y/o SIDA) y sus allegados (1). Actualmente la Ley General sobre VIH-SIDA desde 1998, así como su reforma en el 2008, pretende fortalecer los servicios de atención integral en salud de las personas con $\mathrm{VIH}^{(2)}$. Mediante la atención integral que es aquella multidisciplinaria e integrada que apoya a las personas con VIH y a los miembros de su familia y la comunidad ${ }^{(2)}$. Incluye actividades de promoción de la salud, prevención de la enfermedad, atención de la morbilidad y de las condiciones discapacitantes, atención de la morbilidad, fortalecimiento de la calidad y proyecto de vida, enfoque biopsicosocial, espiritual y ecológico, trabajo inter-transdisciplinario y abordaje intersectorial ${ }^{(2)}$.

\section{DISCUSIÓN}

\section{ATENCIÓN DEL VIH/SIDA}

La atención clínica es el control de la enfermedad y fortalecimiento de la calidad de vida, disminución de la cadena de transmisión epidemiológica (proceso desde la detección temprana de la infección hasta el control y la rehabilitación) ${ }^{(2)}$ En el 2010 se confeccionó el nuevo Plan Estratégico Nacional 2011-2015, que contiene las acciones y estrategias prioritarias que el país busca promover el abordaje integral de la epidemia ${ }^{(1)}$. Sin embargo, llama la atención que sobre el tratamiento, atención y apoyo, no se mencionan los servicios de rehabilitación ${ }^{(1)}$.

CONASIDA es la máxima instancia a nivel nacional encargada de recomendar las políticas y los programas de acción de todo el sector público, relacionados con los asuntos concernientes al VIH y SIDA ${ }^{(1)}$.

También debe coordinar con las diferentes instituciones, fomentando la coordinación y acuerdos interinstitucionales; velando por la plena observancia y respeto de los derechos y garantías de las personas que viven con VIH y SIDA, sus familiares y allegados ${ }^{(1)}$ Está compuesto por el Ministerio de Educación Pública, Ministerio de Justicia y Gracia, Ministerio de Salud, Caja Costarricense de Seguro Social, Universidad de Costa Rica, Organizaciones No Gubernamentales y personas con $\mathrm{VIH}^{(1)}$.

En el Informe Nacional sobre los Avances en la Lucha contra el SIDA del 2012, se evidencia que existen obstáculos administrativos y jurídicos que impiden en el acceso a la atención integral (1). Existe desproporción con respecto al apoyo político de los altos funcionarios de instituciones del Estado y entre instituciones públicas ${ }^{(1)}$.

El apoyo político aún no cuenta con el total compromiso de altas autoridades y se considera que debe fortalecerse ${ }^{(1)}$. Entre otras razones, también se presentan las actitudes estigmatizantes y discriminatorias ${ }^{(1)}$. Los servicios de apoyo complementario que requieren (por ejemplo empleo, acompañamiento a sus familias), son muy incipientes ${ }^{(1)}$. 
VIH- SIDA

La infección por VIH es considerada una enfermedad crónica desde la década de 1990, que se asocia con una amplia gama de condicionesdeficiencias neurológicas, neuromusculares y cardiopulmonares, por lo tanto, a discapacidad (3). Al aumentar la sobrevida-sobrevivencia, aumenta la discapacidad asociada ${ }^{(3,4,22)}$. Las personas con VIH asintomáticas y sintomáticas experimentan niveles más altos de discapacidadincapacidad física, comparadas con la población general $^{(4)}$.

Los principales predictores de expectativa en las personas con VIH son: conteo de CD4, diagnóstico de VIH tardío, inicio de tratamiento antirretroviral (TARV) tardío o adherencia incompleta, estadio de la enfermedad en la que se inicia el TARV, mantenerse o no dentro del sistema del cuidado de la salud ${ }^{(5)}$.

También son importantes los estilos de vida y el comportamiento: tabaquismo, consumo de alcohol, uso de drogas intravenosas, coinfección con hepatitis, enfermedades de transmisión sexual (ETS) ${ }^{(5)}$. Y por último, los factores sociodemográficos: género, edad, comorbilidades asociadas al envejecimiento, etnia o país de origen, lugar de residencia-vecindarios, estatus socioeconómico ${ }^{(5)}$.

\section{CLÍNICA DEL VIH/SIDA}

\section{VIH}

El VIH tiene la habilidad de afectar múltiples sistemas y órganos, especialmente sistema nervioso, tanto central como periférico, siendo asombrosa la variedad de síndromes clínicos ${ }^{(6)}$. Adicionalmente muchas de las infecciones oportunistas y malignidades asociadas, así como el tratamiento médico, pueden alterar la funcionali$\operatorname{dad}^{(6)}$.

Las neuropatías más comunes en personas con VIH son la polineuropatía sensitiva distal dolorosa y la neuropatía tóxica por TARV ${ }^{(4)}$. Las neuropatías secundarias al TARV-neurotóxico altera primero las fibras de bajo calibre. Mientras que en las que se relacionan con el VIH, la alteración es de las fibras de grueso calibre y por disminución de fragmentos de fibras nerviosas ${ }^{(7)}$. Las personas que viven con VIH y además neuropatía periférica relacionada al VIH sensitiva distal, reportan dolor de moderado a severo y trastornos del sueño significativos, así como presentan limitación en las distancias de ambulación $(243.99 \pm 141.04 \text { metros })^{(8) .}$

Las poliradiculopatías agudas en pacientes infectados por VIH se deben principalmente al citomegalovirus $(41 \%), M$. tuberculosis $(18 \%)$, y linfomatosis meníngea y meningitis criptoccóci$\mathrm{ca}^{(9)}$.

El Síndrome de Guillian Barré asociado al VIH es un fenómeno bien documentado, que típicamente ocurre con la seroconversión ${ }^{(10) \text {. }}$

Se consideran hallazgos tardíos la ataxia, debilidad, tremor, mioclonus, convulsiones, incontinencia, mutismo aquinético ${ }^{(11)}$.

La fatiga es una de las quejas más comunes de los infectados por VIH, y de los peores síntomas (12). Puede limitar la calidad de vida de aproximadamente el 54\% de las personas con $\mathrm{VIH}$, predominantemente mujeres ${ }^{(12) .}$ La discapacidad asociada a la fatiga incluye dificultad para el desempeño de actividades físicas críticas como el empleo y manejar, así como actividades instrumentales de la vida diaria ${ }^{(12) .}$ Por lo tanto, la entidad que se ha llamado Desorden Neurocognitivo asociado al VIH (gran espectro de anormalidades cognitivas, motoras y del humor), debe tamizarse, ya que puede mejorar con TARV con buena penetración y actividad en SNC asociado a intervenciones neurorehabilitatorias, es decir, con un manejo multidisciplinario ${ }^{(13) .}$

Las manifestaciones de discapacidad son de naturaleza multidimensional, impredecible y episódica, con dificultades para enfrentar el día a día, desafíos para la inclusión social y la incertidumbre sobre el futuro, por lo tanto el rol de la

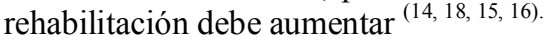

Los profesionales en rehabilitación pueden y deben asistir a las personas con VIH desde el diagnóstico para manejar la discapacidad secundaria a efectos adversos de los medicamentos, fatiga, dolor, neuropatía, problemas cognitivos, así como apoyo vocacional ${ }^{(14,16)}$. Es decir, mantener o aumentar la calidad de vida, minimizar su dependencia funcional y disminuyendo los costos de cuidado de salud, disminuir la comorbili$\operatorname{dad}^{(16) \text {. }}$

El complejo demencial (trastorno de la memoria, pobre concentración, enlentecimiento cognitivo) es el síntoma más frecuente, afectando a un $65 \%$ 
de los pacientes ${ }^{(2)}$. Los dominios que muestran problemas significativos son especialmente la función motora, ejecutiva, velocidad de procesamiento de la información y lenguaje, con un deterioro cognitivo global leve ${ }^{(7)}$. La depresión produce sufrimiento, disminución de la funcionalidad y alteración de la calidad de vida de las personas con $\mathrm{VIH}^{(3)}$.

Al menos el $80 \%$ de los sobrevivientes han experimentado mínimo una limitación/deficiencia, limitación para la actividad o restricción de la participación, atribuible a su estado de portador del VIH en el último mes ${ }^{(15) .}$

Los pacientes en varias etapas de la infección por VIH requieren los servicios de rehabilitación: polineuropatías, disfagia, disfunción autonómica, falla respiratoria, disfunción vesical e intestinal, debilidad generalizada, depresión, por ejemplo ${ }^{(2)}$

Ya que presentan problemas para cada disciplina del equipo de rehabilitación (Terapia respiratoria, física, ocupacional, del lenguaje, entre otros) ${ }^{(2)}$. Muchas de las complicaciones de salud a largo plazo en esta población, pueden ser tratadas exitosamente con fisioterapia ${ }^{(17)}$.

Debido a la mejoría del tratamiento de la infección por $\mathrm{VIH}$, la rehabilitación como rama de la medicina, va a estar más involucrada en el cuidado del paciente con VIH ${ }^{(2) .}$

\section{SIDA}

Las personas con SIDA muestran una alteración aún más significativa si están afectadas la memoria visual, construcción visual, atención, velocidad psicomotora, aprendizaje, coordinación motora, memoria verbal y razonamiento ${ }^{(7) .}$ Los déficit cognitivos se asocian con disminución o deterioro de la funcionalidad diaria y estado vocacional, es decir, en el autocuidado, vida independiente, logros académicos, manejo de medicamentos, finanzas, cocina y compras, por el requerimiento de múltiples habilidades cognitivas para llevarlas a cabo ${ }^{(7)}$.

Por eso también, esta población es propensa al desempleo ${ }^{(7)}$. Aunque los problemas en el funcionamiento cognitivo no son una barrera para el empleo, si no están severamente debilitados ${ }^{(7)}$. Sólo el funcionamiento ejecutivo predice positivamente la actividad laboral ${ }^{(7)}$. Las pruebas neuropsicológicas son la herramienta más útil para predecir el reingreso a la fuerza laboral o para preparar el regreso al trabajo ${ }^{(7)}$.

Pero los factores motivacionales son más predictivos que el funcionamiento cognitivo ${ }^{\text {(7). }}$ Seguido de las polineuropatías sintomáticas, en el 50\% (2). Los cambios conductuales (apatía, depresión) corresponden al $40 \%$, y pueden ser la manifestación de presentación de la infección en el $20 \%{ }^{(2)}$.

En aproximadamente el $20 \%$ de las personas con SIDA presentarán síntomas neurológicos como la manifestación inicial de la enfermedad ${ }^{(11)}$. Un ECV puede ser la primera manifestación de infección por VIH, por lo tanto debe considerarse (descartarse) en pacientes jóvenes (11). La incidencia es de $8-34 \%$ en estudios patológicos y menor al 1-7\% en estudios clínicos ${ }^{(11)}$. Los eventos isquémicos son más frecuentes que los hemorrágicos ${ }^{(11)}$. Los pares craneales más afectados son el V, VI y VII ${ }^{(4)}$.

En general, las complicaciones de SNC son extremadamente comunes en personas con SIDA (11). Los signos neurológicos se documentan en el $90 \%$ de los hospitalizados ${ }^{(11) .}$

Los déficits funcionales frecuentes en pacientes con SIDA hospitalizados son: cognitivos, respiratorios, dolor, fatiga, hemiparesia, los cuales requieren de intervenciones rehabilitatorias (terapia física, ocupacional, del lenguaje, modalidades de medios físicos, dispositivos-ayudas técnicas, medicamentos adicionales, electrodiagnóstico, entre otros) ${ }^{(3)}$.

Un tercio de los pacientes con SIDA después de estar hospitalizados, al egreso terminan con una discapacidad significativa, con dificultad para su movilidad y autocuidado ${ }^{(3)}$. También puede haber anormalidades del asta anterior de la médula y de la unión neuromuscular ${ }^{(18)}$.

\section{REHABILITACIÓN}

Las necesidades básicas de las personas con VIH pueden agruparse en 3 grandes áreas:

1) Servicios de prevención y consejería

2) Servicios de diagnóstico y tratamiento

3) Servicios de rehabilitación: cuidado en casa, soporte psicológico y social, actividades recreativas-entretenimiento- 
grupos de apoyo-redes, soporte nutricional, aseguramiento y empleo ${ }^{(19)}$.

Motivos de referencia frecuentes a rehabilitación de los pacientes con SIDA son:

$-76 \%$ alteración de la movilidad

$-57 \%$ dificultad para autocuidado

$-45 \%$ disfunción neurológica (hemiparesia, cognitiva, demencia, mielopatía, miopatía, neuropatía) $-37 \%$ dolor $^{(6)}$.

Se prefiere el tratamiento no farmacológico del dolor como primera línea de tratamiento si es de leve a moderado, por la gran cantidad de medicamentos requeridos en esta población ${ }^{(18)}$

El fisiatra puede colaborar en un diagnóstico a tiempo, sin procedimientos que sean innecesarios y prevención de dolores iatrogénicos, y garantizar el confort y calidad de vida ${ }^{(20)}$.

$-29 \%$ alteración cognitiva

$-27 \%$ desacondicionamientogeneralizado

-Manejo de úlceras

-Efectos locales del Sarcoma Kaposi

-Síndromes varios musculoesqueléticos

-Ceguera ${ }^{(6)}$.

Las intervenciones de rehabilitación se basan en los déficit funcionales, en vez del progreso de la enfermedad, lo que sugiere que los médicos especialistas en Medicina Física y Rhabilitación tiene el conocimiento adecuado para manejar la mayoría de discapacidad relacionada con el VIH

\section{METÓDOS Y TÉNICAS DE TRATAMIENTO}

Muchas limitaciones-discapacidades, se asocian a la infección por VIH ${ }^{(2)}$. La rehabilitación provee beneficios que mejoran la calidad de vida de las personas infectadas, pero también para la sociedad como un todo ${ }^{(2)}$. Los especialistas en Medicina Física y Rehabilitación deberían tener un rol central dentro de la educación al público y a quienes confeccionan las políticas relacionadas a la atención integral del VIH ${ }^{(2,22)}$. La rehabilitación es la piedra angular para garantizar la calidad de la rehabilitación en VIH ${ }^{(22)}$. Existen responsabilidades legales y éticas relacionadas a la Rehabilitación y la provisión del cuidado de las personas con VIH de una forma humana $\mathrm{y}$ compasiva $^{(2,22)}$.
La comunidad de rehabilitación debe familiarizarse e involucrarse en el proceso de atención de las personas con VIH/SIDA obligatoriamente ${ }^{(18)}$ Asumiendo y explorando los mecanismos necesarios para ofrecer el cuidado compasivo y apropiado de esta población, respondiendo a las necesidades cambiantes ${ }^{(18,15)}$. Desde la promoción y prevención, hasta cuidado a largo plazo y paliativo ${ }^{(15)}$.

Los pacientes con infección por VIH son muy similares-requieren el mismo abordaje por rehabilitación, que los pacientes con cáncer: permanente, individualizada, adaptada, estrechamente vinculada con los demás cuidados médicos, se debe considerar su pronóstico, condición médica actual, necesidades del paciente y su familia y/o cuidadores, determinación de metas funcionales y planeamiento interdisciplinario, es decir, la filosofía general ${ }^{(11,18,12,23)}$. Al ser una enfermedad crónica progresiva, requiere de un manejo médico intensivo en una escala comparable con las malignidades ${ }^{(6)}$

Es un proceso complejo que responde a la aplicación integrada de muchos procedimientos ${ }^{(23)}$. La progresión de la enfermedad se caracteriza usualmente por múltiples exacerbaciones de complicaciones médicas, de las cuales el paciente usualmente se recupera, por lo tanto, el manejo es similar ${ }^{(22) .}$ La metodología de tratamiento y el abordaje de rehabilitación en la persona con VIH debe ser flexible y adaptativo, pretender disminuir los costos y el sufrimiento, y mantener la productividad-funcionalidad tanto como sea posible ${ }^{(6,22,24) \text {. }}$

Se manejan los mismos principios de tratamiento que en pacientes cuyos procesos no se relacionan con $\mathrm{VIH}^{(18)}$

Algunos ejemplos de intervenciones implementadas están:

$-73 \%$ ejercicio terapéutico

$-45 \%$ ayudas para la marcha

$-45 \%$ equipo de seguridad y para el baño

$-29 \%$ ortesis

$-29 \%$ manejo del dolor (TENS, calor, vibración, masaje) ${ }^{(6,18)}$

$-10 \%$ tanque de remolino

$-4 \%$ consejo vocacional ${ }^{(6)}$

Es decir, que los pacientes con SIDA, tienen una gran variedad de déficit físicos y limitaciones funcionales, por lo tanto, requieren de múltiples 
intervenciones de rehabilitación ${ }^{(6)}$. Todos los pacientes con SIDA deberían ser evaluados por un fisiatra, como política estándar del Servicio de Rehabilitación, para minimizar la disfunción temprana y en etapas tardías proveer de un ambiente seguro al paciente ${ }^{(6) .}$

La sensación de control es el "grado de confianza global en uno mismo, de la habilidad a través de amplio rango de situaciones demandadas" (25). Se correlaciona con la adherencia al tratamiento farmacológico, la cual es un factor determinante en los pacientes con tratamiento antiretroviral (TARV), ya que se requiere de un $95 \%$ de adherencia para ser efectivo ${ }^{(25)}$. En la población con el VIH, también disminuye los hábitos poco saludables y aumenta las conductas promotoras dela salud y resultados inmunológicos mejores ${ }^{(25)}$. Es decir, que trabajar para mejorar la sensación de control, mejora la salud en general ${ }^{(25)}$.

El ejercicio es una terapia adyuvante en el manejo de enfermedades crónicas ${ }^{(25) .}$ Puede ser una estrategia importante en la promoción de la salud para mejorar el bienestar psicológico, ya que ha demostrado en estudios de otras poblaciones que podría ser una forma costo-efectiva para prevenir o manejar síntomas depresivos de leves a moderados ${ }^{(3,26)}$. En personas con VIH es beneficioso y seguro: aumenta el acondicionamiento cardiovascular, la fuerza muscular, mejora el ánimo, podría mejorar aspectos metabólicos y morfológicos de la lipodistrofia ${ }^{(23,25,25)}$. Previo a TARV, aumenta la calidad de vida ${ }^{(25)}$. Posterior al TARV, el enfoque se dirige a los efectos adversos de los medicamentos y a mejorar el bienestar $(25,26)$. Los efectos beneficiosos potenciales del ejercicio dependen de una adherencia óptima ${ }^{(25) .}$ Un supervisor experimentado (terapeuta físico con experiencia en población VIH), podría mejorar la adherencia ${ }^{(25)}$ El ejercicio es una estrategia clave para tratar a las personas con VIH ${ }^{(26)}$

El entrenamiento de resistencia de grandes grupos musculares, a una intensidad moderada de $60-80 \%$ de repeticiones máximas (RM) y con un aumento progresivo, mejora la composición corporal y fuerza muscular (aumenta la masa magra, músculo y densidad mineral ósea y disminuye de peso), con impacto limitado en la calidad de vida ${ }^{(23,26) \text {. }}$

El ejercicio aeróbico de moderada intensidad (11-14 en la Escala de Borg o 50-85\% de la Frecuencia cardiaca máxima), mejora la composición corporal (porcentaje de grasa) y capacidad aeróbica ${ }^{(23,26,25)}$.El entrenamiento combinado produce una ganancia en todos los aspectos relacionados con la composición corporal, fuerza muscular, flexibilidad, acondicionamiento cardiopulmonar y habilidad funcional, pero se requiere que sea a una intensidad moderada y de larga duración ${ }^{(23,26,27)}$.

El ejercicio debe ser prescrito por profesionales en rehabilitación y también debe ser supervisado, para minimizar las lesiones y maximizar los beneficios ${ }^{(23)}$. El programa de ejercicio debe hacerse de acuerdo a la función física individual estado de salud, respuesta al ejercicio y metas establecidas ${ }^{(23)}$.

La intensidad y volumen del ejercicio son determinantes importantes en las respuestas fisiológicas al ejercicio ${ }^{(23)}$. No existe un consenso sobre qué modalidad e intensidad son más efectivos en personas con VIH, por lo tanto, es difícil escoger el mejor entrenamiento de la población $(23,26)$. Se requieren más estudios para determinar parámetros óptimos de ejercicio aeróbico para que sea lo más beneficioso para adultos con VIH (26).

Durante la evaluación de la persona con VIH es importante considerar antes de prescribir el ejercicio: el riesgo de caídas, contraindicaciones para el ejercicio (pérdida de peso rápida secundaria a enfermedad aguda o infección(es) oportunista(s) aguda), enfermedad avanzada (CD $4<100 / \mathrm{mm}^{3}$, ya que no es capaz de adherirse al programa de ejercicio prescrito), capacidades limitadas para el ejercicio por afección del sistema nervioso central y periférico y del sistema musculoesquelético, y los efectos adversos del TARV, así como alteraciones del humor y la prevalencia de comorbilidades ${ }^{(11)}$.

La rehabilitación puede contribuir al manejo de los pacientes con SIDA hospitalizados ${ }^{(3)}$. Sólo una minoría de los profesionales en rehabilitación trabajan con personas con $\mathrm{VIH}{ }^{(26)}$. A pesar de que existe evidencia que documenta la discapacidad física en VIH y la necesidad de la población por dichos servicios ${ }^{(14) .}$ En cierto grado, ya que los especialistas en VIH tienden a referir a trabajo social y organizaciones no gubernamentales (ONG) para tratar-abordar deficienciaslimitaciones asociadas al VIH ${ }^{(14) .}$ Los profesionales en rehabilitación tienen dentro de su rol, promover la salud mental de las personas con $\mathrm{VIH}^{(14) \text {. }}$ 
El $85 \%$ de los pacientes consideran que la rehabilitación es importante para las personas con VIH, pero sólo el $19 \%$ consideran que los profesionales en rehabilitación tienen las capacidades y conocimientos adecuados ${ }^{(15) \text {. }}$

El $61 \%$ de los profesionales en rehabilitación nunca han trabajado con personas con $\mathrm{VIH}^{(15) .} \mathrm{Y}$ los que han trabajado con la población, sólo han atendido 4 personas al año ${ }^{(15) \text {. }}$

Sólo el 27\% de los profesionales en rehabilitación han recibido educación sobre VIH como parte de su programa de grado, y sólo el $11 \%$ han participado en un entrenamiento especial o educación continua en el área de VIH/SIDA en los últimos 5 años ${ }^{(15)}$.

Los profesionales en ciencias de la salud, por ejemplo los estudiantes de terapia física y ocupacional, expresan miedo y ansiedad por atender a personas con $\mathrm{VIH}^{(6)}$. Principalmente ligados a su género y creencias religiosas ${ }^{(6)}$. Lo cual puede afectar la evaluación y la calidad de los servicios (6).

Existe un 27\% de los profesionales en rehabilitación que no quieren trabajar con la población y $46 \%$ están inseguros (28). Existe un progreso modesto de la intervención-manejo de las personas con VIH, con un enfoque hacia calidad de vida $^{(18)}$.

Siendo 2 de sus determinantes más importantes los síntomas y el desempeño físico ${ }^{(18)}$. En general, la discapacidad severa en SIDA es inusual, es frecuente e leve a moderada, la más frecuente es en Actividades Instrumentales de la Vida Diaria (AIVD), y la discapacidad no se relaciona con el conteo de CD4 ${ }^{(18)}$.

\section{CONCLUSIÓN}

Como parte del tratamiento de los pacientes con VIH/SIDA, es indispensable la participación de los especialistas en rehabilitación, dando así una atención para mejorar la calidad de vida de los pacientes.

A participación en el proceso de rehabilitación es un trabajo multidisciplinario en donde se trabaja en equipo Médico fisiatra, terapeuta físico $\mathrm{y}$ terapeuta Ocupacional, así como la coordinación con el servicio de infectologia y demás profesionales del la salud. De tal manera que se pueda contribuir al desarrollo de una vida plena, mejorando así los estándares en la calidad de vida.

Dentro de las modalidades de fisioterapia el ejercicio aeróbico, ayudas biomecánicas, agentes físicos, ejercicio terapéutico, entre otras técnicas son funcionales y efectivos para esta población siendo la Fisioterapia y Rehabilitación convencional una opción necesaria para el tratamiento de la población con VIH/SIDA.

\section{BIBLIOGRAFÍA}

1. Ministerio de Salud CONASIDA. Informe Nacional sobre los avances en la lucha contra el sida 2010-2011. 2012; 1-90 y 3-5.

2. Ministerio de salud. Plan estratégico nacional (PEN) VIH y sida 2011-2014. 2010; 1 102 у $3-7$.

3. O Dell M. Rehabilitation Medicine Consultation in Persons Hospitalized with AIDS: an analysis of thirty cases AM J Phys 1993; 72: 90-96.

4. Worthington C Myers T O'Brien K Nixon $\mathrm{S}$,Cockerill R Bereket T. Rehabilitation professionals and humanimmunodeficiency virus care: results of a national Canadiansurvey. Arch Phys Med Rehabil 2008;89:105113.

5. A. Sabin C. Do people with HIV Infection have a normal life expectancy in the era of combination antiretroviral therapy. BMC Medicine 2013: 7-7.

6. O Connell P Levinson S. Experience with rehabilitactionadquiredinmunodeficiencysindrome. Rehabilitation in AID 1991: 70 : 195-199.

7. Chernoff R Martin D Schrock Huy M. Neuropsychological functioning as a predictor of employment activity in a longitudinal study of HIV-infected adults contemplating workforce reentry. Journal of the international neuropsychological society 2010;16:38-48.

8. Sandoval R Roddey T Giordano T Mitchell K Kelley C. Pain, Sleep Disturbances, and FunctionalLimitations in People Living with HIV/AIDS-Associated Distal Sensory Peripheral Neuropathy. Journal of the international association of providers of AIDS care 2007: 1-7.

9. O Brien K Bone G Zack E Solomon P. HIV and rehabilitation: development of conceptual framework for curriculum planning. In- 
ternational Journal of Rehabilitation Research 2008; 31: 189-197.

10. Schreiber AL Norbury J-W Sousa EAFunctional recovery of untreated human immunodeficiency virus associated GuillainBarre' syndrome: A case report. Elsevier Masson SAS. United States.

11. O Dell MW Sasson NL. Hemiparesis in HIV infection: Rehabilitation approach. Am J. Phys Med Rehabil 1992; 71:291-296.

12. Keyser RE Peralta L Cade WT Miller S Anixt J. Functional aerobic impairment in adolescents seropositivefor HIV: a quasiexperimental analysis. Arch PhysMedRehabil 2000;81:1479-84.

13. Chan LG Wong CS. HIV-associated neurocognitive disorders - An issue of growing importance. Ann acad Med Singapore 2013;42:527-534.

14. O Brien K Wilkins A Zack E Solomon P. Scoping the field: identifying Key Research Prioritiesin HIV and Rehabilitation. AIDS Behave 2010; 14: 448-458.

15. O Brien K Bone G Zack E Solomon P. HIV and rehabilitation: development of conceptual framework for curriculum planning. International Journal of Rehabilitation Research 2008; 31: 189-197

16. Levinson SF O Connell PG. Rehabilitation dimensions of AIDS a review. ArhPhys Med Rehabil 199; 72: 690-696.

17. Pullen SD Chigbo NN Nwigwe et all. Physiotherapy intervention as a complementary treatment for people living with HIV/AIDS. HIV/AIDS - Research and Palliative Care 2014:6 99-107.

18. O Dell M. HIV - Related neurological disability and prospects for rehabilitation. Disability and rehabilitation 1996;18:286-292.

19. Moradi G Mohraz M Gouya MM et all. Health Needs of People Living with HIV/AIDS: From the Perspective of Policy Makers, Physicians and Consultants, andPeople Living with HIV/AIDS Iranian J Publ Health 2014;43:1424-1435.

20. Domínguez G Zalaya I Montejo G. Cuidados paliativos en enfermedades infecciosas VIH y Sida, dindromes dolorosos. Tomo 1, capitulo 3: 423-445.

21. O’Dell MW Levinson SF Riggs RV: Physiatric management of HIV-related disability. Arch PhysMed Rehabil1 996; 77: 66-73.

22. Levinson SF O Connell PG. Rehabilitation dimensions of AIDS a review. ArhPhys Med Rehabil 199; 72: 690-696.
23. Hernández MS Maturell J Vigil IA Rodriguez MJ. Actualización de tema Rehabilitación fisioterapéutica de personas viviendo con el virus de inmunodeficiencia humana $y$ sida. Medisan 2011;15: No.9

24. Servicio de atención en salud. Guía de atención del VIH/SIDA. Colombia 2015: 1 -34.

25. Fillipas S Oldmeadow LB Bailey MJ Cherry CL. A six-month,supervised, aerobic and resistance exercise program improves selfefficacy in people with human immunodeficiencyvirus: A randomised controlled trial. Australian Journal of Physiotherapy 2006; 52: $185-190$.

26. O'Brien K Nixon S Tynan AM Glazier R. Aerobic exercise interventions for adults living with HIV/AIDS. CochraneDatabase of Systematic Reviews 2010; Issue 8: 1-73.

27. Keyser RE Peralta L Cade WT Miller S Anixt J. Functional aerobic impairment in adolescents seropositivefor HIV: a quasiexperimental analysis. Arch PhysMedRehabil 2000;81:1479-1484.

28. O Brien K Bone G Sinclare L Solomon P. Rehabilitation in the Context of HIV:AnInterprofessional Multi-Stakeholder Process for Curriculum Development. J Allied Health 2010; 39:131-137.

\section{CONFLICTOS DE INTERES}

Los autores declaran que no existe ningún conflicto de interés. 